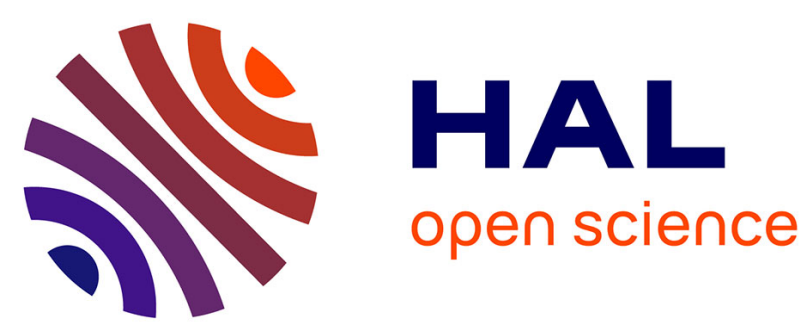

\title{
Terminologie et ontologie
}

Christophe Roche

\section{- To cite this version:}

Christophe Roche. Terminologie et ontologie. Langages, 2005, La terminologie: nature et enjeux, 157. hal-01354691

\section{HAL Id: hal-01354691 \\ https://hal.science/hal-01354691}

Submitted on 19 Aug 2016

HAL is a multi-disciplinary open access archive for the deposit and dissemination of scientific research documents, whether they are published or not. The documents may come from teaching and research institutions in France or abroad, or from public or private research centers.
L'archive ouverte pluridisciplinaire HAL, est destinée au dépôt et à la diffusion de documents scientifiques de niveau recherche, publiés ou non, émanant des établissements d'enseignement et de recherche français ou étrangers, des laboratoires publics ou privés. 


\section{Persée \\ http://www.persee.fr}

\section{Terminologie et ontologie}

\section{Christophe Roche}

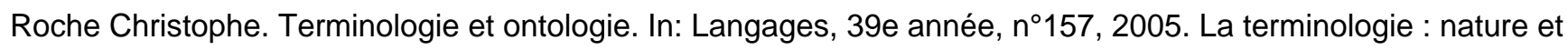
enjeux. pp. 48-62.

\section{Voir l'article en ligne}

\section{Christophe Roche : Terminologie et ontologie.}

Occidental philosophy has been for centuries implicated in the way languages refer to the world. Ontologies are the result of the thought applied to the existence of things. Nowadays, ontologies represent entities handled by companies, such as products, processes, and so on. They are more and more involved in data processing. Actually, one of the aims of ontology treatment is to make sure that a designation corresponds to a concept and that both concept and designation- refer to a well specified object. Logic as science of well formulated propositions is also involved in such data modeling.

\section{Avertissement}

L'éditeur du site «PERSEE »- le Ministère de la jeunesse, de l'éducation nationale et de la recherche, Direction de l'enseignement supérieur, Sous-direction des bibliothèques et de la documentation - détient la propriété intellectuelle et les droits d'exploitation. A ce titre il est titulaire des droits d'auteur et du droit sui generis du producteur de bases de données sur ce site conformément à la loi nº $98-536$ du 1 er juillet 1998 relative aux bases de données.

Les oeuvres reproduites sur le site «PERSEE » sont protégées par les dispositions générales du Code de la propriété intellectuelle.

Droits et devoirs des utilisateurs

Pour un usage strictement privé, la simple reproduction du contenu de ce site est libre.

Pour un usage scientifique ou pédagogique, à des fins de recherches, d'enseignement ou de communication excluant toute exploitation commerciale, la reproduction et la communication au public du contenu de ce site sont autorisées, sous réserve que celles-ci servent d'illustration, ne soient pas substantielles et ne soient pas expressément limitées (plans ou photographies). La mention Le Ministère de la jeunesse, de l'éducation nationale et de la recherche, Direction de l'enseignement supérieur, Sous-direction des bibliothèques et de la documentation sur chaque reproduction tirée du site est obligatoire ainsi que le nom de la revue et- lorsqu'ils sont indiqués - le nom de l'auteur et la référence du document reproduit.

Toute autre reproduction ou communication au public, intégrale ou substantielle du contenu de ce site, par quelque procédé que ce soit, de l'éditeur original de l'oeuvre, de l'auteur et de ses ayants droit.

La reproduction et l'exploitation des photographies et des plans, y compris à des fins commerciales, doivent être autorisés par l'éditeur du site, Le Ministère de la jeunesse, de l'éducation nationale et de la recherche, Direction de l'enseignement supérieur, Sous-direction des bibliothèques et de la documentation (voir http://www.sup.adc.education.fr/bib/ ). La source et les crédits devront toujours être mentionnés. 


\section{Terminologie et ontologie}

«C'est parce que les choses ont une essence que les mots ont un sens "

Pierre Aubenque, Le problème de l'être chez Aristote, 1994

Terminologie et ontologie. Deux termes qui pourraient s'opposer à plus d'un titre - le premier portant sur une pratique, le deuxième relevant de la métaphysique - et qui sont néanmoins de plus en plus associés. Deux termes qui, s'ils partagent une même visée normative, restent à préciser.

Cet article a pour objet de présenter et de situer les principales approches relevant de la terminologie et de l'ontologie et d'indiquer en quoi elles peuvent être liées. Nous verrons en particulier quels peuvent être les apports de l'ontologie, tant d'un point de vue méthodologique que pratique, pour la modélisation et la représentation de la signification des termes. Pour cela nous serons amené à distinguer ce qui relève de l'usage, de l'intellection et de la représentation.

Activité pratique répondant à un besoin de clarification, la terminologie est également une science. Elle constitue une discipline à part entière qui, tout en puisant à la linguistique et à l'épistémologie, s'en distingue.

Plusieurs problèmes ne seront pas abordés dans le cadre de cet article d'introduction générale, aussi importants soient-ils, et même si l'ontologie (ou les ontologies) apporte des solutions novatrices. Nous pensons en particulier au problème de la diachronie et à celui de la mise en correspondance de terminologies " locales " à des communautés de pratique.

Enfin, si des citations émaillent l'article, elles ne sont pas toujours à prendre au pied de la lettre, mais plutôt à entendre comme éléments de réflexion.

Les notes apparaissent dans le corps du texte, à la demande de l'auteur [NdIR]. 


\section{PROLÉGOMÈNES}

\subsection{Le Choix du titre}

«Terminologie \& Ontologie ${ }^{1}$ ». Un tel titre peut surprendre si l'on se place à la fois dans une filiation wüsterienne ${ }^{2}$, même revisitée, de la terminologie et sous une étiquette métaphysique de l'ontologie $^{3}$ comme le suggère la citation de Pierre Aubenque.

Au-delà des positions et des conflits idéologiques, un tel titre trouve sa justification dans des aspirations partagées, même si leurs préoccupations restent différentes ${ }^{4}$, quant à la recherche d'un savoir établi pouvant servir de référence commune et par le rôle que peut jouer le législateur en tant que " créateur de noms ".

Enfin, l'ordre des mots du titre suggère que notre étude se fera sous l'angle des apports de l'ontologie - comprise ici comme démarche, méthode, d'où son emploi au singulier - pour la définition des concepts à la base de la signification des termes.

1 Cet article présente les résultats de nos travaux de recherche menés dans les domaines de l'ingénierie des connaissances, de l'ontologie et de la terminologie. Il prend en compte les retours d'expériences des applications industrielles menées par la société Ontologos Corp. et l'équipe Condillac, Équipe de Recherche Technologique, de l'Université de Savoie. Les applications terminologiques ont été réalisées à l'aide de l'environnement LCW (Linguistics Craft Workbench) pour l'analyse automatique de corpus à des fins d'extraction de candidats termes, la OK Station (Ontological Knowledge Station) et l'atelier OCW (Ontology Craft Workbench) pour la construction de réseaux sémantiques et d'ontologies (ontologies définies par différenciation spécifique) et à l'aide de l'environnement TCW (Terminology Craft Workbench) pour la définition des fiches terminologiques.

Les notes sont placées en fin de chapitre et non en bas de page.

Les mots du lexique situé en fin d'article sont suivis d'un astérisque lors de leur première apparition dans le texte.

2 Les travaux de Wüster se rattachent à ceux du Cercle de Vienne dont un des objectifs est de s'opposer au retour de la métaphysique. Si les ontologies sont effectivement utilisées par Wüster, ce n'est pas pour définir l'être selon son essence, mais pour définir le concept (notion) comme composé d'éléments.

3 C'est-à-dire dans une optique de compréhension des objets du monde. Le même titre surprendrait moins si l'on considérait, selon le point de vue de l'ingénierie des connaissances, l'ontologie comme moyen de représentation des objets du monde à des fins de manipulation.

4 Préoccupations liées à une pratique pour la terminologie, et à la théorie de la connaissance pour l'ontologie.

\subsection{Le sens des mots}

Les mots ont une histoire. Tout au long de leur vie ils se chargent de sens, s'en délestent et en changent jusqu'à parfois aboutir à des contresens.

Ceux de terminologie et d'ontologie ne font pas exception, à un point tel qu'ils peuvent chacun sous un même vocable désigner des théories et des approches différentes, voire opposées. La confusion qui en résulte pour des disciplines ayant une visée normative leur est parfois préjudiciable et est à l'origine de nombreuses incompréhensions.

C'est pourquoi nous replacerons chaque fois que nous le pourrons les mots dans leur histoire et leur contexte sans prétention d'imposer une définition au détriment d'une autre. Mais c'est en précisant celles que nous utiliserons que nous pourrons construire un discours cohérent. 


\subsection{Les pratiques}

Parler de terminologie c'est parler de mots et du sens des mots. Pouvons-nous dire pour autant que la terminologie relève uniquement de la linguistique ? L'extralinguistique, qui de facto s'exclut de la linguistique, et dont relève la conceptualisation du monde, intéresse au premier chef la terminologie.

Souvent les hommes de l'art (au sens du grec "tekhnê ») reprochent à la langue usuelle son manque de précision, tout comme les tenants d'une langue plus naturelle critiquent la rigidité des langues de spécialité. Pouvons-nous parler pour autant d'imperfection des langues ? Ou plutôt de pratiques inappropriées de langues hors de leurs champs d'application?

Les mots "hyper(hypo)onymie ", "subsomption ", " généralisation vs spécialisation " revêtent une importance toute particulière pour le domaine qui nous intéresse. Souvent employés avec une certaine confusion car se référant à la même idée de " sens plus large ", de " contenant " ou d' " englobant ", ils se rapportent pourtant à des domaines et à des pratiques différents. Le premier appartient sans conteste à la linguistique, et à elle seule, et lie des mots entre eux. Le second relève de l'épistémologie (au sens de la théorie de la connaissance) et structure des concepts*. Enfin, le dernier est du ressort de la représentation : nous ne manipulons la réalité qu'à travers les représentations que nous en avons (pour l'intelligence artificielle, existe ce qui peut être représenté). Bien maîtriser ces différences évite des méprises fâcheuses ${ }^{1}$. Doit-on nécessairement décréter qu'une discipline a la préséance sur une autre ? Qui, du langage ou de la pensée, de la pensée ou du réel, précède et conditionne l'autre? Nous sommes davantage en présence d'appréhensions différentes mais complémentaires et indissociables du monde correspondant à des pratiques différentes. L'intérêt du triangle sémiotique "vox - conceptus - res " apparaît ici pleinement, plus qu'en tant que schéma réducteur du sens d'un mot.

Nous avons affaire à trois pratiques différentes pour lesquelles trois langages sont nécessaires.

- La "langue d'usage (ou des usages) ${ }^{*}$, objet de la linguistique, est liée à un usage pratique de l'univers et prend en compte la notion de locuteur.

- La « langue de l'intellection »* est quant à elle liée à la connaissance intel-

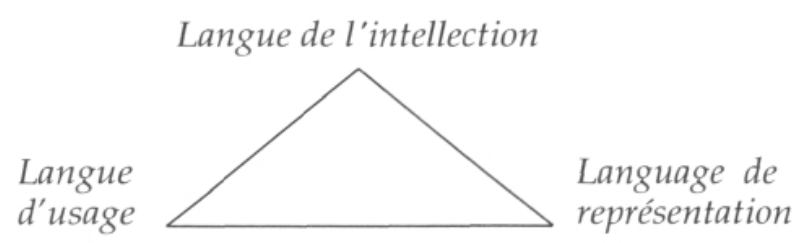
lectuelle que nous, observateurs, avons de l'univers relativement à des principes épistémologiques. Elle se veut indépendante des contingences liées aux usages.

- Les «langages de représentation »* ont pour objectif de représenter les entités du monde réel afin de les manipuler.

La sémantique de ces langages diffère et ne peut être abordée de la même façon ${ }^{2}$.

Une telle approche permet d'affirmer que le signifié et le concept sont distincts et ne relèvent pas des mêmes pratiques, que les langages de représentation ${ }^{3}$ ne définissent ni une théorie de la connaissance ni une langue d'usage.

Si nous devions illustrer, au sens propre, ces différences de pratique, nous prendrions pour exemple deux tableaux de Jan Vermeer. L' " Astronome " figure l'intellection, cette volonté de comprendre le monde et l'importance de l'observateur, la main tendue vers le globe terrestre. Le "Géographe » illustre l'activité du scientifique dans 
sa tentative de construction d'une représentation du monde - construction artificielle et manipulable, ici une carte - le globe rangé au sommet de l'armoire.

1 Si la relation d'hyperonymie peut lier un mot à un ou plusieurs de ses hyperonymes, comme une classe peut, selon les formalismes de représentation utilisés, être liée à une ou plusieurs sur-classes, cela n'est pas nécessairement le cas pour la relation de subsomption (comme par exemple la relation de subsomption reposant sur la définition de l'espèce en genre et différence).

2 Quoi que nous fassions nous ne pouvons sortir de la langue. Ainsi nous avons besoin de mots pour définir les concepts. Mais les mots qui les utilisent et ceux qui les définissent ne relèvent pas du même système. Nous retrouvons la distinction entre langage et métalangage.

3 Les langages de représentation sont principalement des outils dont les principes épistémologiques ne sont pas toujours explicites et clairs. Sachant que in fine nous manipulons une représentation des objets du monde réel, le "danger " est alors de modéliser le monde à l'aide des principes épistémologiques sous-jacents au langage de représentation et de réduire le vocabulaire des mots d'usage aux noms des entités composant la représentation finale.

\title{
2. LA TERMINOLOGIE
}

\begin{abstract}
"Puisque les mots sont les signes de nos idées, il faut que le système des langues soit formé sur celui de nos connaissances. Les langues, par conséquent, $n^{\prime}$ ont des mots de différentes espèces, que parce que nos idées appartiennent à des classes différentes; et elles n'ont des moyens pour lier les mots, que parce que nous ne pensons qu'autant que nous lions nos idées "
\end{abstract}

Etienne Bonnot de Condillac, Grammaire, 1780

\subsection{Définition}

Plus une pratique qu'une théorie - on peut se poser la question de la considérer comme une pratique théorisée - une terminologie peut être vue comme une langue ${ }^{1}$ de spécialité, c'est-à-dire une langue liée à une pratique technique ou scientifique propre à un domaine. Les termes employés sont spécifiques à un métier et renvoient à une réalité extralinguistique partagée : objets (artefacts), pratiques, méthodes, processus, etc. Ces termes n'ont pas le même statut que les mots de la langue usuelle.

Cette pratique est une réponse à un besoin et à une volonté de clarification et de normalisation ${ }^{2}$ des mots et de leur signification ; normalisation de leur signification en dehors de la diversité des usages ${ }^{3}$, normalisation de la construction ${ }^{4}$ du mot luimême devant refléter sa signification.

En cela nous suivons l'étymologie du mot "terminologie ". C'est au XVIII siècle, dans le contexte des projets encyclopédiques et de la pensée qu'une science est avant tout une langue bien faite - selon l'expression de Condillac -, que se développe le besoin d'une description normée de la signification des termes scientifiques et techniques. La terminologie " moderne " prend véritablement son essor dans la première moitié du vingtième siècle à partir des travaux d'Eugen Wüster qui posent les principes méthodologiques du travail terminologique.

La terminologie, étude des mots techniques et de leur signification, est une discipline dont le statut n'est pas clairement reconnu. Entre langue d'usage et langue d'intellection, devant répondre à des besoins pratiques, il lui reste encore à affirmer sa place pour être reconnue pleinement comme discipline à part entière.

1 En toute rigueur, une terminologie n'est pas une langue. Elle s'intéresse à des notions (concepts) et aux expressions les nommant (approche onomasiologique) ou à des expressions métiers et aux connaissances 
qu'elles désignent (approche sémasiologique). L'étude des pratiques langagières liées à une technique ou une science, à travers la rédaction de documents scientifiques ou techniques par exemple, ne relève pas directement de la terminologie mais de la linguistique.

2 Cette idée de normalisation se trouve dans l'étymologie du mot "terme ", du latin "terminus ", qui limite, qui borne.

3 Si un mot n'a peut-être pas de sens hors texte (en considérant que le sens est lié aux usages du mot), il peut avoir une signification hors texte en référence à une réalité extralinguistique ou à un "sens normé ".

4 La position de Cratyle - " on connaît les choses par leur nom " - reste d'actualité en terminologie.

\subsection{Langue d'usage}

Considérant le fait que la terminologie est une langue de spécialité caractérisée par l'usage de mots spécifiques à un métier, et que ces mots constituent les seules données observables dont nous puissions disposer, il semble naturel de baser la construction de la terminologie sur le traitement des matériaux qui les contiennent, à savoir les textes ${ }^{1}$ scientifiques et techniques.

Une analyse linguistique des corpus rend possible une extraction " automatique " de syntagmes définissant autant de candidats termes soumis à la validation des experts du domaine.

Les termes ainsi obtenus, même liés entre eux par des relations linguistiques telles que l'hyperonymie ou la méronymie, ne constituent pas une terminologie. Il reste à leur associer une signification.

La sémantique distributionnelle - c'est-à-dire l'analyse de la distribution de mots sur la base de contextes ${ }^{2}$ - nous aide à construire la signification des termes en demeurant au plus près des textes. Les champs lexicaux que la sémantique distributionnelle permet de déterminer apportent une aide précieuse pour la définition et l'organisation des concepts.

Cette démarche sémasiologique aboutit à la construction de terminologies qui dépendent du corpus choisi avec pour conséquence une "ré-utilisabilité " qui peut être faible, si ce n'est dans les mots métier, du moins dans les concepts associés. Certains diront alors - en dehors de tout phénomène diachronique - qu'une terminologie est propre à une application donnée.

Mais cela est-il surprenant? Il n'y a pas de concepts dans un texte, mais uniquement des traces linguistiques de leurs usages. Nous modélisons ici le sens des mots observés en discours, c'est-à-dire leur signifié. Une telle démarche relève de la lexicologie et non plus de la terminologie - rappelons que la linguistique se fonde principalement sur la relation entre le signifiant et le signifié alors que la terminologie s'intéresse en premier à la relation entre le concept et l'objet. De surcroît, comment prendre en compte la diversité des productions textuelles ${ }^{3}$ pour en dégager un sens commun, objet de la terminologie, alors que les différents niveaux de discours ne peuvent qu'entraîner des perturbations notionnelles et désignationnelles ${ }^{4}$ ?

Cette critique ne remet pas en cause l'intérêt de la sémantique distributionnelle, bien au contraire, l'analyse linguistique de corpus constitue une des premières étapes, quasiment incontournable, de la démarche terminologique. Elle nous permet simplement de dire que la terminologie n'est pas uniquement une langue d'usage.

1 En fait, les textes sont les seuls objets d'observation. Les mots observés le sont en discours, au sein de textes; ce qui nous amène à nous poser la question de savoir si ce que nous modéliserons sera la signification* des mots ou leur sens* (signifié). 
2 Recherche de co-occurrences, étude des candidats termes générés à partir de patrons lexico-syntaxiques (étude des expressions avec une même extension ou un même préfixe), étude du rôle des adjectifs dans la construction de syntagmes nominaux, etc.

3 Une étude de la typologie des textes (descriptifs, informatifs, prescriptifs, définitoires, narratifs, etc.) et de leur structure montre bien la diversité des usages et tout l'intérêt qu'il y a à la fois à tenir compte des textes mais aussi à être capable de s'en détacher.

4 À un point tel que parfois il est utile de revenir à une démarche plus "classique " basée sur l'analyse des axes syntagmatique et paradigmatique des entretiens avec les experts du domaine.

\subsection{Sens et signification}

Il convient de distinguer le sens* d'un mot de sa signification*. Le sens d'un mot - ou signifié - variable au sein d'un même discours, se définit par rapport à ses usages. Son étude relève de la linguistique. Le signifié peut être défini de différentes façons pourvu que cette définition reflète les usages du mot. Définition par rapport à un système qui peut être la langue elle-même, définition en langage naturel ou définition par rapport aux autres mots en indiquant en quoi ils diffèrent ${ }^{1}$; ou par rapport à un système formel comme l'est un système conceptuel.

La signification d'un mot se veut, quant à elle, indépendante des usages du mot. Elle peut être définie en référence à un "sens normé ", sens commun à plusieurs discours, si on veut rester en langue, ou en référence à une réalité extralinguistique comme l'est une conceptualisation du monde. Sa définition est faite dans un système qui peut être la langue elle-même, définition en langage naturel, ou dans un système formel, par exemple conceptuel.

Nous pouvons maintenant également définir le sens d'un mot comme étant une signification actualisée en discours ${ }^{2}$.

1 Sémantique structurale (le mot a une valeur plus qu'un sens [F. de Saussure]) et analyse sémique sont des exemples de tels systèmes qui considèrent la langue comme un système de mots organisés différentiellement les uns par rapport aux autres.

2 Il existe des mots, en particulier techniques, dont l'interprétation n'est pas conditionnée par le contexte; tout comme nous pouvons concevoir des syntagmes ayant un sens mais pas de signification.

\subsection{Langue de l'intellection}

"Il convient de se rappeler que tout travail terminologique devrait être fondé sur des notions et non sur des termes"

Helmut Felber, Manuel de Terminologie, 1984

La terminologie, en tant que science des termes, est à la fois une activité pratique dans sa volonté de compréhension des objets du monde et de clarification des discours et une science qui repose sur la distinction entre la langue et l'extralinguistique.

La terminologie ne s'intéresse aux mots que dans la mesure où ils désignent des notions ${ }^{1}$ (ou concepts). Un terme ${ }^{* 2}$ est donc une entité à double face, combinaison indissociable d'un nom - une désignation* - et d'un concept.

Au même titre que la langue peut être considérée comme un système, la terminologie est un système de termes reflétant une modélisation conceptuelle. Le modèle conceptuel, plus généralement dénommé "système notionnel ", est donc au cœur de toute terminologie et trouve sa raison d'être dans la façon dont nous appréhendons les objets du monde. La terminologie est une langue de l'intellection qui étudie les rapports entre les concepts et les choses. 
En tant que modélisation conceptuelle, le système notionnel est extralinguistique, ce qui ne veut pas nécessairement dire interlinguistique. Il peut l'être pour des communautés de pratique dont les langues d'usage partagent la même conceptualisation du monde. La terminologie gagnerait à ne plus être perçue comme une discipline purement normalisatrice au service d'une pensée unique. Il semble plus réaliste de dire qu'une normalisation traduit la reconnaissance d'une intersubjectivité partagée au sein d'une communauté de pratique. Il devient alors possible de parler de "terminologies régionales " et de prendre en compte les particularismes linguistiques et conceptuels. La compréhension (traduction) entre différentes communautés peut être abordée sous l'angle d'une "mise en correspondance » de systèmes notionnels différents.

En tant que langue de l'intellection, l'approche terminologique est principalement onomasiologique et les concepts doivent être, dans la mesure du possible, nommés de telle sorte que les noms reflètent la structure notionnelle. L'organisation du système notionnel et les principes épistémologiques sur lesquels repose la terminologie ont donc un impact jusqu'à la dénomination* des concepts. La définition des concepts en genre et différence est une des plus "belles " illustrations de cette approche (une presse hydraulique est une sorte de presse dont le piston est mû hydrauliquement). Le vocabulaire, ici la liste des désignations, ainsi construit est normé et considéré comme sans ambiguïté (monosémique).

Cependant il est important de rappeler que les mots d'usage (qui peuvent être polysémiques) doivent être pris en compte, mis en relation avec les désignations du vocabulaire normé, et leurs différents contextes d'utilisation précisés.

1 L'usage du mot "concept" semble plus adapté que celui de "notion" en particulier lorsque nous faisons référence à la théorie supportant la modélisation du système notionnel. Un "bel » exemple qui montre que nous ne pouvons faire fi des mots d'usage, même pour parler de terminologie.

2 Comme le signe est la combinaison indissociable d'un signifiant et d'un signifié.

\subsection{Propriétés}

Une terminologie est également un produit, résultat d'une pratique industrielle, et à ce titre doit satisfaire à un certain nombre de propriétés, conditions nécessaires à sa réelle utilisation. Elle se doit d'être, dans la mesure du possible et selon les besoins exprimés, consensuelle au sens où l'on s'accorde sur la définition de ses termes, cohérente, précise dans ses définitions et évolutive dans ses systèmes notionnel et désignationnel.

Ces propriétés dépendent directement des fondements théoriques sur lesquels repose la construction du système notionnel. Une approche scientifique de la signification des termes permet d'atteindre de tels objectifs.

\section{LA MODÉLISATION ET LA REPRÉSENTATION DES SIGNIFICATIONS}

\subsection{Une approche scientifique de la signification}

L'approche scientifique de la signification des termes est une des caractéristiques de la terminologie. Elle consiste à " sortir » de la langue usuelle afin de définir la signification en référence à une théorie formelle, ce qui nous permet de bénéficier des avantages des méthodes hypothético-déductives : l'acceptation de leurs postulats nous oblige à accepter de facto leurs constructions, ici la définition des concepts. 


\subsection{Le concept}

La signification des termes et l'organisation du système notionnel dépendent directement de la théorie du concept qui fonde la terminologie.

Lié à la façon dont nous appréhendons les objets du monde réel, le concept, élément de pensée, est une connaissance portant sur une pluralité de choses distinctes répondant à une même loi. Il correspond à une définition intensionnelle de la classe (ensemble) de ses référents.

\section{i. Ensemble de caractères communs}

La définition du concept comme l'expression d'un ensemble de caractères communs - conditions nécessaires et suffisantes - aux objets d'une même classe est une démarche naturelle et bénéficie d'une longue histoire. Cette première théorie, de tradition aristotélicienne, repose sur l' " axiome des relations internes " qui considère le monde comme un ensemble de substrats supports d'attributs.

La relation de subsomption ${ }^{1}$ organise les concepts par abstraction de caractères communs pour aboutir à une hiérarchie de concepts correspondant à une organisation taxonomique des objets. La manière dont s'effectue la recherche des caractères communs, guidée par la perception ou par la raison, n'est pas sans conséquence sur l'organisation des concepts.

ii. Formule logique

Une tradition logiciste ${ }^{2}$ plus récente élabore en particulier deux autres théories qui ouvrent de nouvelles perspectives. La première, rattachée à la logique extensionnelle des classes, considère le concept comme un prédicat, ou comme une fonction propositionnelle ${ }^{3}$ dont $\mathrm{l}^{\prime}$ argument est un objet et dont la valeur de vérité exprime l'appartenance de l'objet aux référents du concept. La deuxième, liée à la théorie des relations, se fonde sur $\mathrm{l}^{\prime}$ " axiome des relations externes " et permet l'expression de phénomènes relationnels que ne peut pas prendre en compte la logique aristotélicienne "sujet-prédicat ". Les relations mérologiques ${ }^{4}$ - qui s'intéressent à la définition d'un tout en ses parties constituantes - jouent un rôle particulièrement important dans la description des objets.

Les concepts - formules bien formées de la logique - peuvent se définir comme conjonction ou disjonction, voire négation, de concepts existants ${ }^{5}$.

1 Un concept A subsume un concept B si et seulement si l'intension de B contient l'intension de A ou inversement si l'extension de A contient l'extension de B.

2 "Logiciste " désigne ici un programme de représentation du concept sur une base logique.

3 Ainsi, si « Homme » est un concept, "est mortel " est également un concept.

4 Du grec méros, partie. La "Méréologie » est un calcul des "totalités et de leurs parties". Voir en particulier les travaux de Stanislaw Lesniewski qui ont abouti à une logique qui lui est propre : $l$ " "Ontologie".

5 Que l'on peut également interpréter dans le cadre de la théorie des ensembles appliquée aux extensions des concepts (classes).

\subsection{La représentation}

Les langages de représentation de connaissances issus de l'intelligence artificielle sont parmi les formalismes les plus utilisés. C'est en particulier le cas des systèmes à base de schémas qui offrent une représentation " externe " lisible. Les schémas (classes) sont définis par un ensemble d'attributs communs à leurs instances (exemplifications), et se structurent selon une relation hiérarchique de spécialisation. Nous 
avons ici affaire à des outils - non à un support méthodologique pour la représentation des connaissances - dont l'objectif est la représentation d'entités manipulables.

Les langages logiques jouent également un rôle important dans la mesure où ils offrent un cadre théorique garant a priori des propriétés recherchées. Cependant la logique, depuis qu'elle est mathématique, n'est plus épistémologique, ce qui pose un certain nombre de problèmes ${ }^{1}$. La logique est nécessaire, mais elle l'est a posteriori au sens où la représentation formelle du système notionnel doit pouvoir donner lieu à une interprétation logique. Elle ne doit pas l'être a priori au sens où la modélisation du monde ne peut être guidée par des principes logicistes.

1 D'un point de vue épistémologique un concept n'est pas une formule bien formée de la logique. Un concept est une primitive logique : il n'est ni vrai ni faux, il est. Il subsume des objets de même nature et ne traduit pas la même connaissance qu'un ensemble qui regroupe des objets d'états similaires. Si un concept possède une sémantique ensembliste, tout ensemble n'est pas un concept. Enfin, les prédicats monadiques peuvent aussi bien représenter une connaissance primitive, un concept, qu'un état d'un objet, connaissances pourtant de nature différente.

Une représentation formelle des systèmes notionnels offre de nombreuses perspectives pourvu que les choix épistémologiques aient été judicieux. L'étude des problèmes interlinguistiques à travers l'étude de leurs représentations conceptuelles en est un exemple.

\section{L'ONTOLOGIE}

"Tous les phénomènes contiennent quelque chose de permanent (substance) considéré comme l'objet lui-même, et quelque chose de changeant, considéré comme une simple détermination de cet objet, c'est-à-dire d'un mode d'existence de l'objet "

Emmanuel Kant, Critique de la Raison Pure, 1781

La conceptualisation du monde et sa représentation nous amènent directement à la notion d'ontologie. Cette dernière constitue aujourd'hui une des voies les plus prometteuses quant à la modélisation et à la représentation formelle du système notionnel des terminologies.

Bien que les ontologies aient une visée normative, nous devons faire face à de nombreuses définitions et approches différentes et parfois contradictoires. Les ontologies connaissent aujourd'hui un engouement sans précédent en particulier en ingénierie des connaissances au risque de voir s'imposer le point de vue de cette discipline au détriment de travaux épistémologiques pourtant fondamentaux.

Dans le cadre de ce chapitre nous ne considérerons pas l'ontologie au sens de la terminologie wüsterienne pour laquelle la définition du concept est du domaine de la logique ; l'ontologie ne relève ni de l'épistémologie ni de la métaphysique et on parle davantage de "rapports ontologiques ". Ces "rapports ontologiques » sont définis comme des « rapports indirects entre les notions » dont le type le plus important est la relation mérologique (partitive).

\subsection{Ingénierie des connaissances}

Apparues outre-atlantique au début des années 1990 pour des projets d'ingénierie collaborative ${ }^{1}$, les ontologies connaissent depuis un succès qui ne se dément pas et qui s'explique principalement par la poursuite d'un mythe, celui d'une représentation du monde et du sens des mots pour en parler qui soit compréhensible, et donc partageable, aussi bien par des acteurs humains que par des agents logiciels. 
Comme les terminologies, les ontologies en ingénierie des connaissances sont une réponse à un besoin relativement similaire, celui de la normalisation des communications et des échanges: on ne peut communiquer et partager des informations que si l'on s'accorde un tant soit peu sur les mots utilisés et leur signification. On postule ici que la signification des mots repose sur une représentation partagée et consensuelle d'une modélisation du domaine d'application. Cette représentation constitue une ontologie, à laquelle on associera un vocabulaire de mots constitué des noms des concepts. L'ontologie est l'équivalent du système notionnel et on comprend tout l'intérêt des ontologies de l'ingénierie des connaissances pour les terminologies.

La définition que nous proposons synthétise la plupart des définitions existantes : "Définie pour un objectif donné et un domaine particulier, une ontologie est pour l'ingénierie des connaissances une représentation d'une modélisation d'un domaine ${ }^{2}$ partagée par une communauté d'acteurs. Objet informatique défini à l'aide d'un formalisme de représentation, elle se compose principalement d'un ensemble de concepts définis en compréhension, de relations et de propriétés logiques ".

En tant que produits informatiques à finalité applicative, il est demandé aux ontologies, de façon similaire aux terminologies, de vérifier les mêmes propriétés de consensus, de cohérence, de précision et d'évolutivité afin qu'elles puissent être acceptées et exploitées. Ces propriétés dépendent directement des propriétés du formalisme de représentation utilisé.

Les formalismes les plus utilisés sont ceux issus de la représentation des connaissances en intelligence artificielle - en particulier les systèmes à base de schémas pour leur lisibilité externe. Ce sont également les langages issus de la logique - telles que les logiques des descriptions - qui permettent de garantir certaines des propriétés visées, principalement la cohérence, et qui offrent des opérations intéressantes telle que la classification automatique de concepts.

Les ontologies de l'ingénierie des connaissances ressemblent aux bases de connaissances de l'intelligence artificielle des années 1980. Elles s'en distinguent principalement sur trois points : la recherche de propriétés logiques, la volonté de partage et la recherche de formats lisibles et d'interchange.

Cependant, si in fine c'est bien un objet informatique que les applications terminologiques exploiteront, le problème de sa construction demeure, les mots pour en parler ne font pas l'objet d'études particulières et les propriétés de consensus et d'évolutivité sont rarement atteintes. Les ontologies en ingénierie des connaissances n'ont pas pour objectif de comprendre le monde mais d'en représenter les objets à des fins de manipulation informatique : ce sont des "langages de représentation ». Elles relèvent d'un programme logiciste dans la construction des connaissances sur une base logique et d'un programme mécaniste dans la représentation d'entités manipulables.

Une démarche méthodologique reposant sur des principes épistémologiques clairs est indispensable. C'est la raison d'être première de l'ontologie comme " langue de l'intellection".

1 L'emploi de l'expression "ingénierie collaborative » en lieu et place de "ingénierie simultanée " ou "ingénierie concurrente " illustre bien l'évolution des pratiques en mettant l'accent sur la nécessaire communication et le partage de connaissances entre les différents acteurs collaborant à la réalisation d'un même objectif, comme la conception et la fabrication de produits.

2 En ingénierie des connaissances, une ontologie désigne un objet manipulable et non une discipline. On parle généralement d'ontologies au pluriel ou d'une ontologie au singulier avec un article indéfini. Bien qu'il existe certaines ontologies à visée universelle, les ontologies sont en général propres à un domaine d'application, on parle dans ce cas d'ontologies de domaine, voire à une tâche donnée (ontologies de tâche). 


\section{2. Étymologie}

Bien que de création " récente ", le mot " ontologie " ${ }^{1}$ désigne la science première et relève donc de la métaphysique. Créé au moyen du grec 'to on' à partir du participe présent substantivé du verbe être 'einai' et des mots 'logia' (théorie) et 'logos' (discours), l'ontologie est la «science de l'être en tant qu'être ".

La notion d' " être » a donné lieu à un nombre considérable d'études dont nous ne retiendrons pour notre propre travail (conscient que notre approche est réductrice et simplificatrice) que deux aspects selon que notre intérêt porte sur la compréhension de ce qu'est la chose ou sur sa description telle qu'elle est perçue.

1 Généralement attribué à Christian Wolff "Philosophia prima sive ontologia " (1729), mais antérieurement sous sa forme grecque à Goclenius (1613) et Calov "Scientia de ente " (1636), puis sous sa forme latine à Clauberg (1656) et Réginald (1669).

\subsection{Phénoménologie}

Décrire l'objet tel qu'il nous apparaît, sans spéculation d'aucune sorte, est une démarche naturelle. Ce retour aux choses repose sur la croyance de sens commun qu'il existe des propriétés concrètes, directement accessibles, attachées aux objets. Ces propriétés sont en relation à travers un même substrat, nécessaire mais jamais défini.

L'ontologie est alors la " science de ce qui existe " ${ }^{1}:$ on ne cherche pas à expliquer le monde, mais à le construire sur l'empirie et la logique.

Les objets sont décrits tels qu'ils sont perçus, à travers des qualités que l'expérience leur attribue. Les concepts se forment et se structurent par abstraction de qualités communes. Le choix des qualités communes est arbitraire - la présence conjointe de qualités n'est fondée sur aucun principe explicite ${ }^{2}$ - et peut donner lieu à une multitude de concepts. Si les qualités, issues de l'expérience commune, constituent un médium suffisamment partagé pour servir de référence à une même communauté, ce n'est pas le cas des concepts ainsi construits.

La représentation des ontologies " phénoménologiques " s'appuie principalement sur les langages de représentation issus de l'intelligence artificielle (les qualités correspondent à des attributs valués), la logique et la théorie des relations.

1 Plus que la "science de l'étant en tant que réalisation concrète d'un être ", cette dernière définition supposant un être antérieur à sa réalisation concrète : «la choséité de la chose » (Heidegger).

2 En fait elle repose néanmoins sur une conceptualisation préalable et implicite, que la théorie ne sait ici traduire, comme le montre le choix de noms communs comme dénominations des concepts.

\subsection{Métaphysique}

"Depuis les temps les plus reculés, plus que toute autre branche du savoir, la philosophie a eu le plus d'ambition et atteint le moins de résultats "

Bertrand Russell, La méthode scientifique en philosophie, 1914

En tant que science première, une définition étymologiquement plus juste du mot " ontologie " est : " la science de l'être en tant qu'être indépendamment de ses déterminations particulières ". Une telle définition relève de la métaphysique. Notre objectif est ici de comprendre en quoi une telle discipline, si fortement critiquée par les scientifiques, peut être utile.

"Comprendre l'être indépendamment de ses déterminations particulières ", c'est vouloir dépasser la description phénoménologique des objets, condition nécessaire 
pour en atteindre une description "stable ". Notre adhésion à un programme aristotélicien s'arrête à cet objectif. En effet, ce n'est pas la recherche de propriétés « objectives " et intrinsèques à l'objet qui font ce qu'il est indépendamment de tout observateur qui nous guide ; mais la recherche de propriétés qui font ce que l'objet est pour une communauté d'observateurs par delà la pluralité de ce qu'il peut être. Le programme ontologique est donc un programme à circonscrire au sein d'une communauté de pratique : il a pour objectif une modélisation ontologique d'une intersubjectivité.

La notion de qualité ou d'attribut, relevant du phénomène et traduisant une connaissance soumise " au plus et au moins ", n'est plus l'ossature sur laquelle se construit l'ontologie. Il nous faut rechercher ce qui définit l'essence de l'objet, ces " caractères eidétiques* " qui font que lorsque l'un d'entre eux est retranché de l'objet, il n'est plus ce qu'il est. Un tel caractère ne peut être valué, c'est sa présence qui est essentielle et non une hypothétique valeur. L'objet concret et ses qualités ne sont plus le sujet premier de notre étude, la perception cède la place à la raison pour l'étude des substrats.

Un concept se définit non plus comme un ensemble de qualités communes à des objets, mais comme un ensemble de caractères eidétiques - dont la présence conjointe n'est pas arbitraire - décrivant sa nature. Ces caractères définissent mais aussi différencient les concepts et les situent les uns par rapport aux autres en tant qu'éléments constitutifs d'un tout.

Les caractères eidétiques, contrairement aux qualités, ne sont pas donnés à l'observation, mais sont issus de l'étude des concepts et de leurs rapports. La relation de subsomption se fonde ici sur la nature des concepts et non sur l'abstraction de qualités perçues.

Enfin, à la définition eidétique d'un concept s'ajoute la donnée des qualités communes aux objets qu'il subsume.

La définition d'un concept par différenciation spécifique - définition en genre et différence - est un exemple de définition basée sur la nature des concepts, les différences étant des caractères eidétiques. Les concepts se structurent alors en un arbre de Porphyre, ossature sur laquelle se grefferont les différentes qualités (attributs ou accidents).

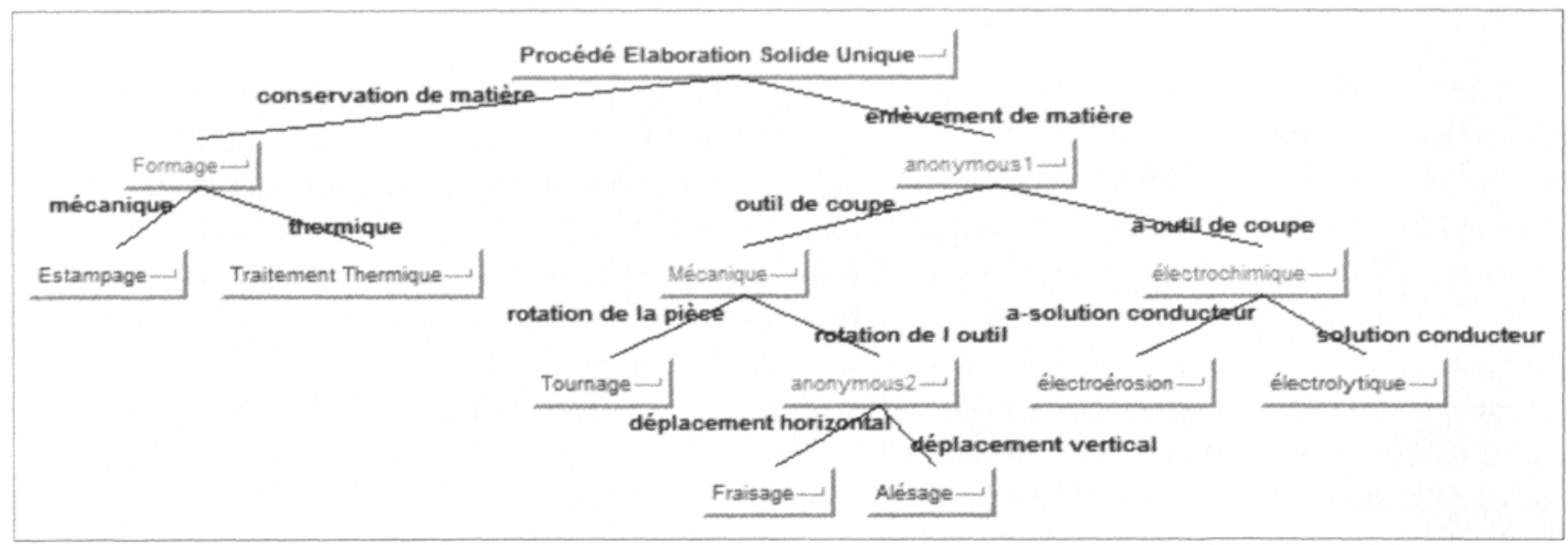

L'approche eidétique, approche à la fois définitionnelle et différenciatrice des concepts, satisfait les propriétés recherchées et aboutit à une méthode de construction des ontologies. Ainsi, la priorité est donnée à l'essence des choses et non à leur état, et la subsomption épistémologiquement fondée guide la construction de l'ontologie. Les principes épistémologiques sont clairs : concept et ensemble ${ }^{1}$ ne se confondent pas et 
il en est de même pour les caractères eidétiques et les qualités ${ }^{2}$. Les définitions sont consistantes au sens logique du terme ${ }^{3}$ et la recherche du consensus s'effectue non pas sur la définition des concepts mais sur l'identification des caractères eidétiques sur laquelle il est plus aisé de s'accorder ${ }^{4}$.

Enfin, la construction des noms des concepts (les désignations) reflète la structure de l'ontologie (vocabulaire orthonormé) et un modèle computationnel ${ }^{5}$ peut être directement construit ainsi qu'une formalisation logique a posteriori.

Cette approche, si elle peut être qualifiée de métaphysique dans sa recherche d'une connaissance au-delà des apparences, demeure néanmoins scientifique par sa définition même et dans la mesure où elle aboutit non seulement à une méthode de modélisation des objets du monde mais aussi à une représentation formelle des objets à des fins de manipulation ${ }^{6}$.

1 Un concept porte sur l'essence des choses et un ensemble sur leur état. Un concept possède bien une interprétation ensembliste (sa définition extensionnelle) alors que l'intension d'un ensemble ne correspond pas obligatoirement à un concept.

2 Le "caractère eidétique", issu de la raison, est essentiel à la définition de l'objet et ne peut être valué. L'attribut - qualité ou accident - participe à la description de l'état de l'objet, il est perçu et valué. Contrairement au caractère eidétique, il n'est pas nécessaire à la compréhension de l'objet.

3 En référence à l'ensemble des "caractères eidétiques " qui constitue, au sens mathématique, une base orthonormée.

4 Ce qui importe n'est pas tant le nom du concept que l'ensemble des caractères qui le définit. Cette démarche n'est pas sans rappeler la théorie atomiste en linguistique basée sur des atomes de sens.

5 Un tel modèle offre des propriétés intéressantes. Par exemple, nous passons d'une complexité exponentielle $\left(2^{n}\right)$ à une complexité linéaire (n) pour la recherche de concepts.

6 Ce qui ressemble fort à un critère de "vérifiabilité" au sens de J. Ayer - ce qui ne manque pas d'ironie ("Langage, vérité et logique ", A.J. Ayer, Flammarion 1956).

Il est important de rappeler que si l'objet nous est donné à travers la perception que nous en avons, il est également pensé ${ }^{1}$. C'est de l'union de ces deux approches, et non de leur exclusion, que naît la connaissance que nous, observateur, avons de l'objet.

1 Nous retrouvons la distinction : phénomène (chose perçue) - noumène (chose pensée).

\subsection{Ontologies textuelles}

L'expression « ontologies textuelles (linguistiques) » est parfois utilisée pour désigner les représentations conceptuelles construites à partir de textes. Elle peut surprendre dans la mesure où l'ontologie, en tant que langue de l'intellection, relève de l'extralinguistique. S'il est vrai que l'étude des textes, en particulier s'ils sont techniques et s'ils reposent sur l'utilisation de langages contrôlés, peut apporter des informations utiles quant à la construction des ontologies, il est également vrai que la langue d'usage "voile » davantage qu'elle ne révèle la nature ontologique des choses - il suffit de penser aux problèmes que soulève la copule "être " pour s'en convaincre -. "Tout n'est pas dans les textes » : les caractères eidétiques sont rarement présents à l'observation ordinaire et ne se laissent pas saisir par des mots d'usage. Les ontologies ainsi construites souffriront des mêmes critiques que celles que nous avons émises concernant l'approche sémasiologique de la terminologie.

Pour conclure ce chapitre, nous dirons qu'il est utile de considérer l'ontologie à la fois comme une méthode de conceptualisation puisant à l'épistémologie, et comme le résultat de l'application de cette méthode à un domaine aboutissant à une représentation particulière relevant de l'ingénierie des connaissances. 


\section{CONCLUSION}

La terminologie est à la fois une pratique et une science. Elle relève de la linguistique par la prise en compte des langues d'usage et s'en distingue par la modélisation scientifique de la signification des mots. Elle relève de l'épistémologie par la recherche d'une compréhension du monde et s'en distingue par l'importance qu'elle donne aux termes, combinaisons indissociables d'une désignation et d'un concept.

Les propriétés de consensus, de cohérence et d'évolution auxquelles doit répondre une terminologie dans le cadre de sa pratique dépendent directement des qualités du système notionnel sur lequel elle repose. L'ontologie, en tant que méthode de modélisation et résultat de son application à un domaine, constitue une des voies parmi les plus prometteuses pour la construction et la représentation du système notionnel des terminologies. Ses différentes approches, qu'elles soient épistémologiques, logiques ou issues de l'ingénierie des connaissances, loin de s'opposer, peuvent ici se compléter et ouvrir des possibilités nouvelles, que ce soit pour l'évolutivité des systèmes notionnels ou leur mise en correspondance dans le cadre de terminologies régionales.

En plus des désignations normées dans leur forme et leur signification (monosémique), il faut également prendre en compte, sans les confondre, les mots d'usage car la terminologie est avant tout une pratique.

Bien que la terminologie puise à diverses disciplines et qu'elle revête une telle multitude de formes qu'il est parfois plus exact de parler de terminologies au pluriel, elle constitue néanmoins une discipline à part entière. Il lui reste à être reconnue en tant que telle.

\section{Bibliographie}

Plutôt qu'une longue liste de publications de recherche, ce genre d'articles appelle davantage des suggestions de lecture en référence à un nombre réduit d'ouvrages. C'est pourquoi nous nous sommes limité seulement à une dizaine de livres en regrettant de laisser de côté de nombreux ouvrages méritants.

Pour la terminologie :

La terminologie, Maria Teresa Cabré, Armand Colin, 1998

Entre signe et concept : éléments de terminologie générale, Loïc Depecker, Presses Sorbonne Nouvelle, 2002

Manuel de terminologie, Helmut Felber, Paris, Unesco, 1984

Travail terminologique, NF ISO 704, AFNOR, avril 2001, ISSN 0335-3931

La terminologie, noms et notions, Alain Rey, PUF, Que sais-je ? $n^{\circ} 178,2^{\mathrm{e}}$ édition corrigée, 1992

Les écrits sur les ontologies sont nombreux, de la métaphysique, en particulier Aristote ( $L a$ Métaphysique) à la phénoménologie, par exemple Heidegger (Qu'est-ce qu'une chose ?), en passant par la rupture kantienne (Kant, Critique de la raison pure). Citons également :

Isagogè de Porphyre, traduction de J. Tricot ou d'Alain de Libera, Vrin

Le problème de l'être chez Aristote, Pierre Aubenque, PUF, Quadrige 1994

Science et métaphysique chez Kant, Michel Meyer, PUF, Quadrige 1988

L'être et l'essence, Étienne Gilson, Vrin, 1994

Critique de la raison pure, Emmanuel Kant, PUF, Quadrige $3^{e}$ édition, 1990

Cours d'étude pour l'instruction du Prince de Parme, Tome Premier, Grammaire, Etienne

Bonnot de Condillac, Genève, chez Du Villard Fils \& Nouffer, 1780

Quant à l'ingénierie de la connaissance en relation avec nos sujets de préoccupations, outre les nombreux articles de recherche, citons :

Sémantique et recherches cognitives, François Rastier. PUF, 1991

Knowledge Representation, John F. Sowa, Brooks/Cole, 2000 
Complétons cette première liste par quelques ouvrages dont la lecture, même si les opinions peuvent fortement différer de ce que nous avons exposé, est des plus profitables :

Signification et nécessité, Rudolf Carnap, Gallimard, 1997

Logique : la théorie de l'enquête, John Dewey, PUF, 1993

Sémiotique et philosophie du langage, Umberto Eco, PUF, 1993

Sémantique structurale, Algirdas Julien Greimas. PUF, 1986

Empirisme logique, Pierre Jacob. Les Éditions de Minuit, 1980

La sémantique du prototype, Georges Kleiber. PUF 1990

Trois essais sur le sensible, Michel Malherbe, Vrin, 1991

Le sens en terminologie, Henri Béjoint et Philippe Thoiron (dir.), ouvrage collectif. Presses universitaires de Lyon, 2000

Représentation et réalité, Hilary Putnam, Gallimard, 1990

Écrits de logique philosophique, Bertrand Russell, PUF 1989

La méthode scientifique en philosophie, Bertrand Russell, Éditions Payot \& Rivages, 2002

\section{Lexique}

(les mots du lexique sont suivis d'un astérisque lors de leur première apparition dans le texte)

caractère eidétique $\quad$ : i. caractéristique liée à l'essence d'un objet

ii. primitive participant à la définition d'un concept

concept

: élément de pensée portant sur une pluralité de choses distinctes répondant à une même loi

dénomination

: nom d'un concept de la terminologie issu d'un processus de nomination (approche onomasiologique) - synonyme : nomination

désignation : mot désignant un concept (approche sémasiologique)

langue de l'intellection : langue liée à la connaissance intellectuelle des objets du monde. Relève de l'extralinguistique

langage de représentation : formalisme de représentation des objets du monde à des fins de manipulation.

langue d'usage

lexicologie

: langue liée à un usage pratique des objets du monde. Relève de la linguistique

nomination

: partie de la linguistique qui s'occupe de la sémantique lexicale; étude des mots et de leur sens

notion

ontologie

: nom d'un concept de la terminologie issu d'un processus de nomination (approche onomasiologique) - synonyme : dénomination

: mot utilisé par la normalisation internationale comme synonyme de concept

: 1. Philosophie

\subsection{Métaphysique}

Science de l'être en tant qu'être indépendamment de ses déterminations particulières

1.2 Phénoménologie

i. Science de l'étant en tant que réalisation concrète d'un être

ii. Science de ce qui existe

2. Ingénierie des connaissances

Représentation d'une modélisation d'un domaine sous la forme d'un ensemble de concepts définis par intension, de relations et de propriétés logiques

sens

signifié

signification

signification d'un mot actualisée en discours

sens d'un mot

système notionnel

1. Terminologie : concept dénoté par une dénomination (désignation)

2. Linguistique: sens normé

terme

: ensemble de concepts sur lequel repose la signification des termes d'une terminologie

: élément d'une terminologie. Combinaison indissociable d'un concept et d'une dénomination (désignation)

terminologie

: science des termes. Étude scientifique des systèmes notionnels (systèmes de concepts) et des mots ou expressions les désignant propres à un domaine

terminologie régionale

: terminologie propre à une communauté de pratique 